\title{
Ajuste de modelos empíricos de infiltración en un Umbrisol bajo diferentes tratamientos silvícolas
}

\section{Fitting of empirical models of infiltration in an Umbrisol under different silvicultural treatments}

Erik Orlando Luna Robles ${ }^{1 *}$, Israel Cantú Silva ${ }^{1}$, María Inés Yáñez Díaz ${ }^{1}$, Humberto González Rodríguez ${ }^{1}$, José Guadalupe Marmolejo Monsiváis ${ }^{1}$ y Silvia Janeth Béjar Pulido ${ }^{1}$

\begin{abstract}
The objective of this research was to model the infiltration process based on results obtained from measurements in situ in different silvicultural stands on an Umbrisol soil in La Ciudad a communal area in Pueblo Nuevo, Durango. Infiltration rate was measured by using the double ring infiltrometer method, for a time of 270 minutes with variable recharges. Post-harvest regeneration silvicultural stands (Clear cutting, Seed-trees and Selection) were analyzed by comparing them with a regenerated area (post-fire) and a reference stand (control). Three empirical models were adapted to 15 infiltration tests that were: Kostiakov, Horton and Lewis-Kostiakov models. The results show an average decrease of $45 \%$ in the initial infiltration $(F i)$, with respect to the Reference stand, the basic infiltration $(F b)$ slight increases in Seed-trees and Selection Trees while Clear cutting and Post-fire reductions of 28 and $23 \%$, respectively. In relation to cumulative infiltration $(\mathrm{Fa})$, only Seed-trees showed $4.7 \%$ above Reference stand. The Analysis of Variance showed significant differences for Fi, while the Kruskal Wallis test showed it for $\mathrm{Fb}$ and $\mathrm{Fa}$. The results of the model fit, based on the coefficient of determination $\left(R^{2}\right)$, show that Lewis-Kostiakov is the model that best estimates the infiltration for the Selection and Reference stands, whose nature is not as affected as the stands of Seed-trees, Clear cutting and Post-fire, which are best described by the Kostiakov type model.
\end{abstract}

Keywords: Seed-trees, clear cutting, model Lewis-Kostiakov, post-fire, infiltration, Umbrisol.

\section{Resumen}

La presente investigación tuvo como objetivo modelar el proceso de infiltración a partir de los resultados de mediciones in situ en diferentes rodales silvícolas en un suelo Umbrisol, localizados en el ejido La Ciudad, Pueblo Nuevo, Durango, México. La infiltración se realizó mediante el método del infiltrómetro de doble anillo, por un tiempo de 270 minutos con recargas variables. Se analizaron rodales silvícolas después de las cortas de regeneración (Árboles Padre, Matarrasa y Selección) y se compararon con un área regenerada (posincendio) y un rodal de Referencia (testigo). Se hizo el ajuste de los datos de 15 pruebas de infiltración a los modelos Kostiakov, Horton y Lewis-Kostiakov. Los resultados mostraron un decremento medio de $45 \%$ en la infiltración inicial (Fi), respecto al rodal de Referencia, la infiltración básica $(F b)$ incrementos ligeros en Árboles Padre y Selección; mientras que Matarrasa y Posincendio presentaron reducciones de 28 y $23 \%$, respectivamente. En relación con la infiltración acumulada $(\mathrm{Fa})$, solamente, el tratamiento de Árboles Padre registró $4.7 \%$ por arriba del rodal de Referencia. El análisis de varianza evidenció diferencias significativas para $F i$; la prueba de las demostró para $F b$ y Fa. Los ajustes de los modelos, basados en el coeficiente de determinación $\left(R^{2}\right)$, mostraron que el de LewisKostiakov estima mejor la infiltración para las áreas de Selección y Referencia, la cual es menos afectada en comparación con los rodales de Árboles Padre, Matarrasa y posincendio forestal, los que fueron mejor descritos por el modelo tipo Kostiakov.

Palabras clave: Árboles Padre, matarrasa, modelo Lewis-Kostiakov posincendio, infiltración, Umbrisol.

Fecha de recepción/Reception date: 21 de agosto de 2019

Fecha de aceptación/Acceptance date: 28 de noviembre de 2019

${ }^{1}$ Facultad de Ciencias Forestales, Universidad Autónoma de Nuevo León, México.

*Autor para correspondencia; correo-e: eranroka@hotmail.com 


\section{Introducción}

Los bosques templados son ecosistemas importantes para la recarga de los mantos acuíferos, ya que se estima que estos proveen $25 \%$ del total nacional que es de 4.8 billones de metros cúbicos de agua (Torres y Guevara, 2002). Al respecto, la Sierra Madre Occidental del estado de Durango es una región vital para las cuencas del océano Pacífico y del interior del centro norte del país (Dueñez et al., 2006).

La capacidad de infiltración es el término aplicado al proceso de entrada de agua a través de la superficie del suelo (Hillel, 1971). La estimación de la velocidad de infiltración y la lámina acumulada es de gran importancia dado que constituyen componentes clave del ciclo hidrológico; dicho cálculo permite cuantificar la escorrentía, la erosión, la disponibilidad de sedimentos, la capacidad de recarga de acuíferos, así como definir la operación de sistemas de riego y estudiar los efectos de diferentes prácticas de uso de la tierra (Grego y Vieira, 2005; Machiwal et al., 2006).

Los usos del suelo asociados con actividades antrópicas, como la agricultura, el pastoreo y manejo forestal pueden afectar negativamente a la biodiversidad y la provisión de servicios ecosistémicos y contribuyen, de manera significativa, en los procesos de cambio climático a nivel regional (Sahagún-Sánchez y Reyes-Hernández, 2018). Además, ocasionan la disminución de las aportaciones hídricas, debido a que el suelo pierde su capacidad de infiltración y de recarga de los acuíferos (Turnbull et al., 2010; Pérez et al., 2018).

El manejo forestal involucra la ejecución de un programa de prácticas silvícolas como son los métodos de regeneración: árboles Padre, corta de selección, cortas sucesivas y cortas totales o matarrasa; así como, todas las actividades de cultivo o tratamientos intermedios entre los que se consideran los aclareos, reforestaciones, quemas prescritas, cortas de saneamiento y podas, que se llevan a cabo durante la gestión del bosque (Monárrez et al., 2018). Dichas prácticas son cuestionadas cuando, por la alta intensidad del aprovechamiento y de las operaciones de abastecimiento, reducen la vegetación forestal; provocan disturbios que exponen el suelo superficial a los efectos de las lluvias, alteran y 
modifican sus propiedades, reducen su capacidad de infiltración e incrementan el riesgo de pérdida de suelo por erosión hídrica (Dueñez et al., 2006).

Pérez y Romance (2012) indican que para conocer el proceso de infiltración se consideran modelos que se ajustan a las mediciones de campo, debido a las diferentes condiciones de suelo, clima, vegetación y manejo. Para estimar la infiltración se han desarrollado modelos matemáticos, los cuales, en general, se dividen en tres grupos (Collis, 1977; Pérez y Romance, 2012): 1) teóricos, 2) semiempíricos, y 3) empíricos. Estos últimos fueron los empleados para la modelación de la infiltración en el presente estudio; ya que no consideran los factores que intervienen en el proceso de infiltración: la textura, el contenido de humedad o la temperatura del suelo, entre otros.

El estudio tuvo como objetivo modelar el proceso de infiltración a partir de mediciones in situ realizadas con la metodología del infiltrómetro de doble anillo, las cuales se ajustaron a los parámetros de los modelos de Kostiakov, Horton y de Lewis-Kostiakov (Weber y Apestegui, 2016), mediante el análisis de su funcionamiento en cinco diferentes rodales silvícolas (tres cortas de regeneración, un área regenerada posincendio y un rodal de referencia) en bosques templados de Durango, México.

\section{Materiales y Métodos}

\section{Área de estudio}

Las pruebas de infiltración se llevaron a cabo en los bosques del ejido La Ciudad, ubicado en el macizo montañoso Sierra Madre Occidental dentro del municipio Pueblo Nuevo, Durango, donde la vegetación dominante está integrada por Pinus durangensis Martínez, Pinus cooperi C.E. Blanco, Pinus ayacahuite Ehrenb. ex Schltdl., Juniperis deppeana Steud. y Quercus sideroxyla Bonpl. (González-Elizondo et al., 2012). El suelo predominante pertenece al tipo Umbrisol (Inegi, 2005) (Figura 1). La precipitación media anual es de $1200 \mathrm{~mm}$, y la temperatura media anual de $18{ }^{\circ} \mathrm{C}$, con máxima de $22^{\circ} \mathrm{C}$ y mínima de $3^{\circ} \mathrm{C}$ (Zúñiga et al., 2018). 


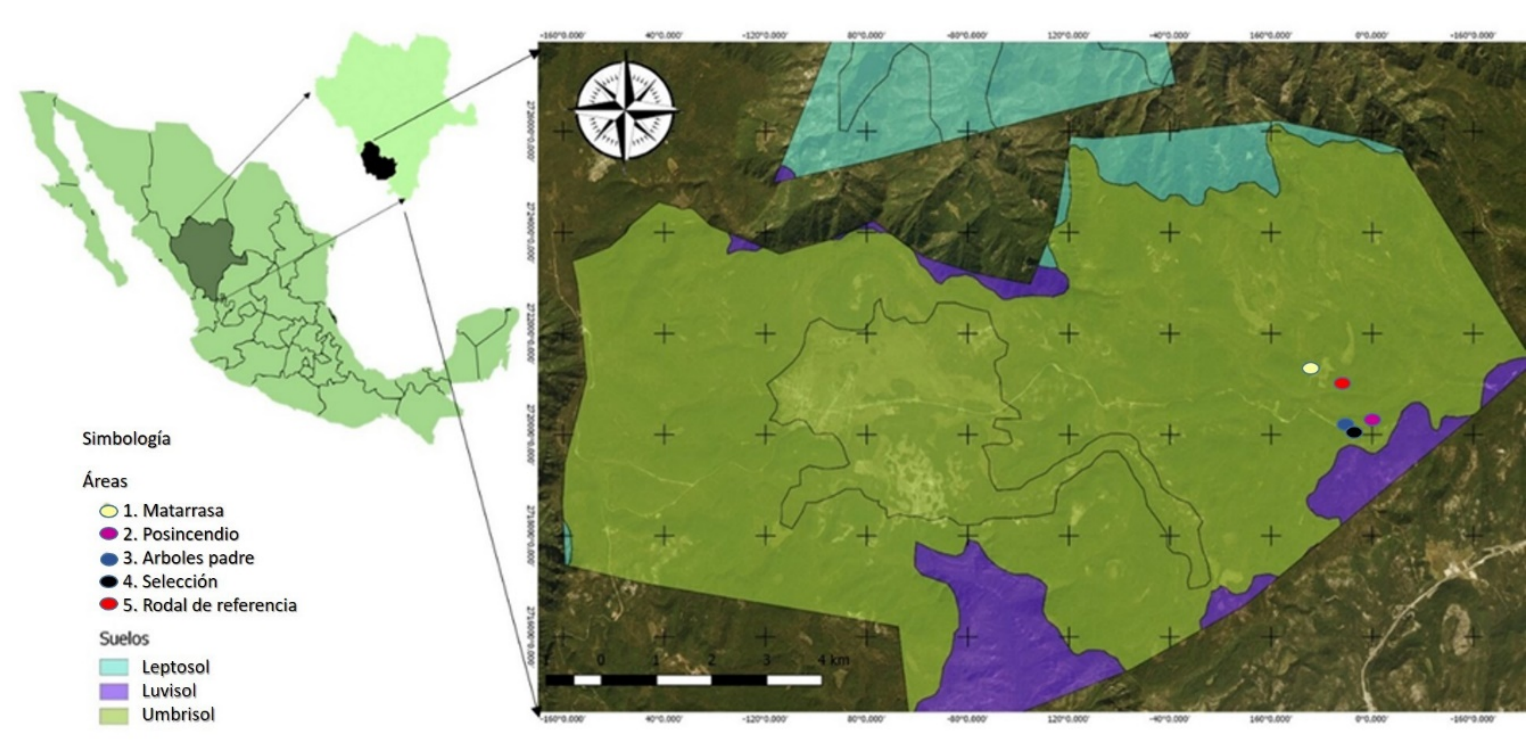

Figura 1. Área de estudio.

\section{Diseño experimental}

La evaluación se realizó en cinco sitios con diferentes condiciones silvícolas que corresponden a tres tipos de cortas, un área regenerada posincendio y un testigo denominado rodal de Referencia. Las áreas de los tratamientos bajo estudio son rodales aprovechados mediante cortas de regeneración con una edad promedio de ocho años, los cuales se describen a continuación: 1) Matarrasa: tiene una superficie de 10.29 ha y consistió en la extracción total del arbolado; 2) Árboles Padre: área de 9 ha y se aplicó una intensidad de corta de 80 \%; 3) Selección: superficie de 20 ha, con un aprovechamiento equivalente a $34 \%$ del volumen total; 4) Posincendio: área regenerada de 10 ha; y 5): rodal de Referencia o testigo de 4.35 ha.

En cada tratamiento (rodal) se aplicaron tres pruebas de infiltración durante la última semana de enero de 2019; para ello se usó un infiltrómetro de doble anillo (metálico), con diámetro interior de $15 \mathrm{~cm}$ y $30 \mathrm{~cm}$ de exterior y una altura de $45 \mathrm{~cm}$; el cual se enterró a una profundidad de $10 \mathrm{~cm}$. Las lecturas se llevaron a cabo por un lapso de 270 minutos, por medio de la aplicación de rellenos (recargas) variables. El proceso 
de medición consistió en la limpieza de la hojarasca presente, enterramiento de los cilindros, se introdujo una regla en el cilindro interior, se colocaba un plástico y sobre este se vertía el agua, al igual que entre los cilindros para prevenir el flujo lateral. Iniciada la prueba se hicieron registros (en $\mathrm{cm}$ ) por minuto durante cinco minutos, cada cinco minutos hasta completar la primera hora de evaluación; en la segunda hora, las mediciones se efectuaron cada diez minutos; en la tercera hora, a los quince minutos; y dos últimas lecturas a los treinta y sesenta minutos, para un total de 270 minutos de evaluación (4.5 horas). Cuando el nivel del agua llegaba a $8 \mathrm{~cm}$, se recargaba el cilindro interior, depositando el agua lentamente; el cilindro exterior se rellenaba cuando disminuía su nivel. Cabe señalar que antes del inicio de las pruebas de infiltración se extrajeron muestras de suelo para determinar el contenido de humedad gravimétrica (Woerner, 1989).

Algunas características del suelo y la vegetación de los sitios se muestran en el Cuadro 1 , ya que son variables de importancia en los procesos hidrológicos.

Cuadro 1. Valores promedio de las características de la vegetación y suelos de los diferentes rodales bajo estudio.

\begin{tabular}{|c|c|c|c|c|c|c|}
\hline \multirow{2}{*}{ Características } & \multicolumn{4}{|c|}{ Vegetación } & \multicolumn{2}{|c|}{ Suelo } \\
\hline & Densidad & DN & Altura & DC & DA & PHA \\
\hline Rodal & (individuos ha ${ }^{-1}$ ) & $(>7 \mathrm{~cm})$ & $(\mathbf{m})$ & $(\mathrm{m})$ & $\left(\mathrm{g} \mathrm{cm}^{3}\right)$ & $(\mathrm{cm})$ \\
\hline Matarrasa & 160 & 7.72 & 3.21 & 1.92 & 0.51 & 1.05 \\
\hline Árboles Padre & 80 & 38.16 & 18.2 & 7.34 & 0.72 & 2.29 \\
\hline Selección & 250 & 26.88 & 15.4 & 5.26 & 0.80 & 3.31 \\
\hline Posincendio & 6400 & 8.13 & 7.42 & 1.33 & 0.58 & 7.00 \\
\hline Rodal de Referencia & 660 & 21.25 & 16.23 & 5.30 & 0.58 & 5.70 \\
\hline
\end{tabular}

DN = Diámetro normal, $\mathrm{DC}=$ Diámetro de copa, $\mathrm{DA}=$ Densidad aparente, PHA = Profundidad de hojarasca acumulada. 


\section{Velocidad de infiltración}

Con los datos de campo se calculó la velocidad de infiltración (Zhang et al., 2017; Yáñez-Díaz et al., 2019) mediante la ecuación:

$$
I=\frac{H L \times 10 \times 60}{t}
$$

Donde:

$I=$ Velocidad de infiltración $\left(\mathrm{mm} \mathrm{h}^{-1}\right)$

$H L=$ Diferencia entre lecturas $(\mathrm{cm})$

10 = Factor de conversión de $\mathrm{cm}$ a mm

60 = Factor de conversión de minutos a horas

$t=$ Período de tiempo $(\min )$

La infiltración acumulada $(F a)$ se determinó mediante la suma total de los volúmenes de agua infiltrada, considerada como la integral de la velocidad de infiltración en esos periodos:

$$
F a=\int_{0}^{t} I(t) d t
$$




\section{Ajuste de los modelos}

Una vez obtenida la curva de infiltración por prueba, se realizó el ajuste de los modelos. Para estimar los parámetros de estos se utilizó el método de mínimos cuadrados (no lineales), que minimiza los errores de ajuste del modelo con la herramienta Solver disponible en la planilla de cálculo Microsoft Excel (Weber y Apestegui, 2016).

Los modelos ajustados para estimar la tasa de infiltración fueron los siguientes:

Modelo tipo Kostiakov (Rodríguez-Vásquez et al., 2008). Se expresa con la ecuación:

$$
I=a t^{b}
$$

Donde:

$I=$ Tasa de infiltración $\left(\mathrm{mm} \mathrm{h}^{-1}\right)$ en un tiempo dado

$t=$ Tiempo (minutos)

$a$ y $b=$ Parámetros de ajuste, el primero está asociado a la infiltración inicial y el segundo a la tasa de cambio

Modelo tipo Horton. Corresponde a un modelo de tres parámetros: Fb, Fi y $K$ (Weber y Apestegui, 2016):

$$
F(t)=F b+(F i-F b) e^{-K t}
$$

Donde:

$F(t)=$ Tasa de infiltración $\left(\mathrm{mm} \mathrm{h}^{-1}\right)$ en un tiempo dado

$F i=$ Tasa inicial de infiltración inicial $\left(\mathrm{mm} \mathrm{h}^{-1}\right)$

$F b=$ Tasa básica de infiltración $\left(\mathrm{mm} \mathrm{h}^{-1}\right)$

$K=$ Parámetro que representa una razón de cambio

$t=$ Tiempo (minutos) 
Modelo tipo Lewis-Kostiakov. Modificación del modelo original de Kostiakov, el cual adhiere a su fórmula el valor de infiltración básica, calculada a partir del promedio de los valores de la tasa para los últimos tres instantes $(180,210$ y 270 minutos) de la prueba de infiltración (Yáñez-Díaz et al., 2019):

$$
F(t)=F b+a t^{-b}
$$

Donde:

$F(t)=$ Tasa de infiltración $\left(\mathrm{mm} \mathrm{h}^{-1}\right)$ en un tiempo dado

$F b=$ Tasa básica de infiltración $\left(\mathrm{mm} \mathrm{h}^{-1}\right)$

$a=$ Parámetro asociado a la infiltración inicial $\left(\mathrm{mm} \mathrm{h}^{-1}\right)$

$t=$ Tiempo (minutos)

$b=$ Parámetro de ajuste

\section{Análisis estadísticos}

Las variables hidrológicas infiltración inicial $(F i)$, infiltración básica $(F b)$ e infiltración acumulada ( $\mathrm{Fa}$ ), así como la humedad gravimétrica fueron sometidas a la prueba de Kolmogorov-Smirnov (Romero, 2016) para verificar los supuestos de normalidad y homogeneidad de varianzas. La variable Fi cumplió con estos, por lo que se le realizó un análisis de varianza para determinar la existencia de diferencias significativas entre los diferentes rodales, con un nivel de significancia de $p<0.05$. A las variables de humedad gravimétrica, Fa y Fb se les aplicó la prueba no paramétrica de KruskalWallis (Berlanga y Rubio, 2012) para determinar la existencia de diferencias significativas $(p<0.05)$ de cometer error tipo I. Los datos se analizaron con el paquete estadístico Statistical Package for the Social Sciences, versión 22 (IBM, 2013). 


\section{Resultados}

Los valores promedios de infiltración inicial, tasas de infiltración básica e infiltración acumulada para los diferentes rodales se muestran en el Cuadro 2, en el que se observa que el rodal de Referencia (testigo) presentó un mejor comportamiento hidrodinámico; caso contrario al tratamiento de Matarrasa, cuyos valores fueron los más bajos en Fi, Fb y Fa. La variable de Fi registró diferencias significativas, por lo que se analizó mediante la prueba de Tukey; Fb y Fa evidenciaron diferencias significativas de acuerdo a la prueba de Kruskal Wallis.

Las pruebas de comparación permiten observar diferencias altamente significativas entre el tratamiento de Referencia y los otros rodales analizados, principalmente, en la variable $\mathrm{Fi}$, la cual se redujo $45 \%$; mientras que la $\mathrm{Fb}$ se incrementó ligeramente en los tratamientos Árboles Padre y Selección, a diferencia de los de Matarrasa y Posincendio en los que se determinaron decrementos de 28 y $23 \%$, respectivamente. En tanto que, $\mathrm{Fa}$, en todos los rodales se redujo, a excepción del área de Árboles Padre la cual registró 4.7 \% por arriba del rodal de Referencia. El contenido de humedad inicial del suelo entre los rodales evidenció diferencias significativas (prueba de Kruskal Wallis, $\mathrm{p}<0.05$ ); por lo que se considera que tiene un papel importante en las condiciones iniciales y finales de la infiltración.

Cuadro 2. Valores medios de las variables hidrológicas en los diferentes tratamientos.

\begin{tabular}{|c|c|c|c|c|}
\hline Tratamiento & $\begin{array}{c}F i \\
\left(\mathrm{~mm} \mathrm{~h}^{-1}\right)\end{array}$ & $\begin{array}{c}F b \\
\left(\mathrm{~mm} \mathrm{~h}^{-1}\right)\end{array}$ & $\begin{array}{c}F a \\
(\mathrm{~mm})\end{array}$ & $\begin{array}{c}H \\
(\%)\end{array}$ \\
\hline Matarrasa & $1000^{a}$ & 214.09 & 10904.95 & 56.23 \\
\hline Árboles Padre & $1350^{b}$ & 299.56 & 17203.79 & 77.70 \\
\hline Selección & $1080^{a b}$ & 322.08 & 15224.30 & 51.52 \\
\hline Posincendio & $1120 \mathrm{ab}$ & 226.95 & 11724.95 & 36.12 \\
\hline Rodal de Referencia & $2080^{c}$ & 297.93 & 16428.29 & 76.61 \\
\hline
\end{tabular}

$F i=$ Infiltración inicial, $F b=$ Infiltración básica, $F a=$ Infiltración acumulada, $H=$ Humedad gravimétrica. Letras distintas indican diferencias significativas (Tukey, $p=0.05$ ). 
La Figura 2 describe el comportamiento de la tasa de infiltración observada en los distintos rodales. En general, se aprecian tres periodos distintos: inicialmente la velocidad de infiltración es alta y se mantiene constante por un corto tiempo (<15 min); después la taza de infiltración baja considerablemente (15$60 \mathrm{~min})$; lo que resulta en un tercer momento de estabilización, denominado tasa de infiltración básica, el cual tiende a apreciarse en todos los tratamientos después de los 200 minutos de aplicación de la prueba.

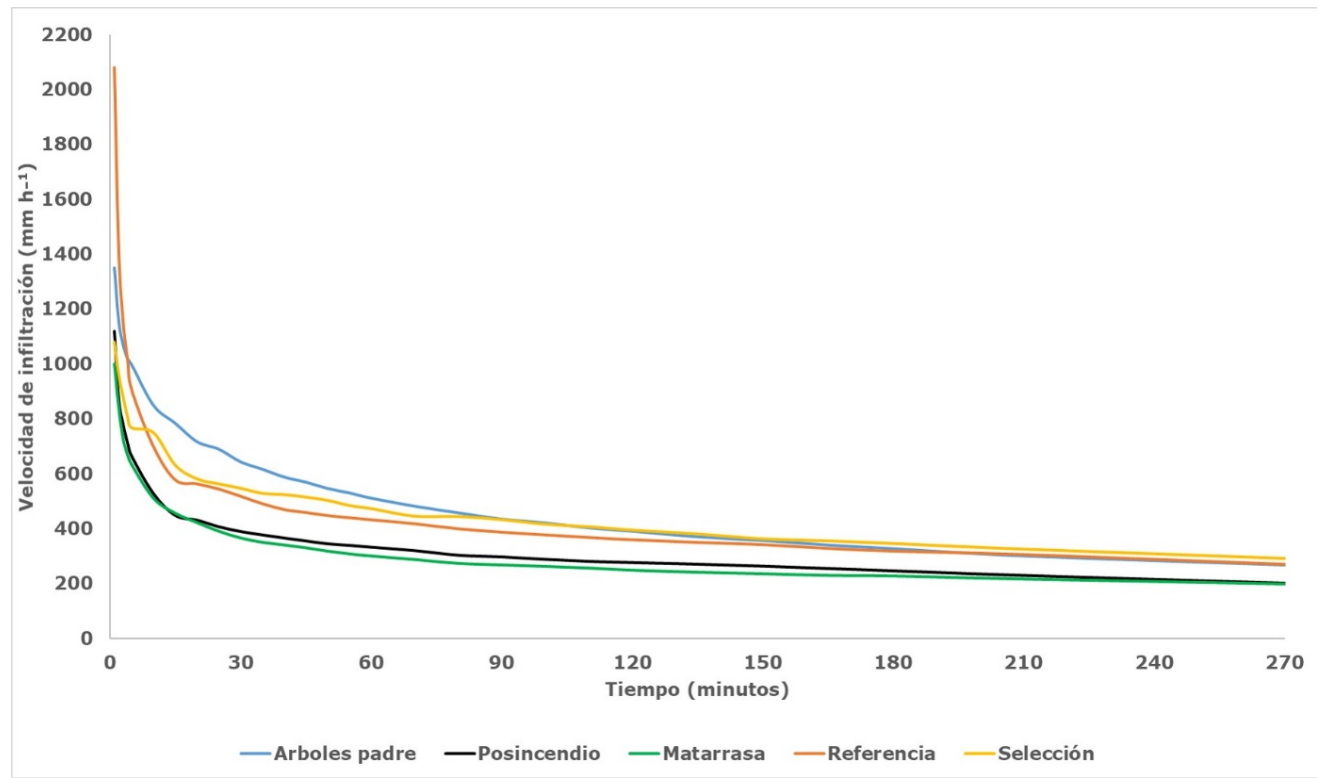

Figura 2. Tasa de infiltración observada en los rodales silvícolas.

En el proceso de infiltración acumulada se demostró que los rodales de Árboles Padre y Referencia tuvieron los mayores volúmenes de infiltración, seguidos del área de aprovechamiento por Selección, el área de posincendio y por último, la de Matarrasa. La infiltración acumulada creció en forma constante durante los primeros 60 minutos, posteriormente empezó a tener un crecimiento con segmentos más estables, la infiltración fue más lenta y prolongada a partir de los 200 minutos (Figura 3). 


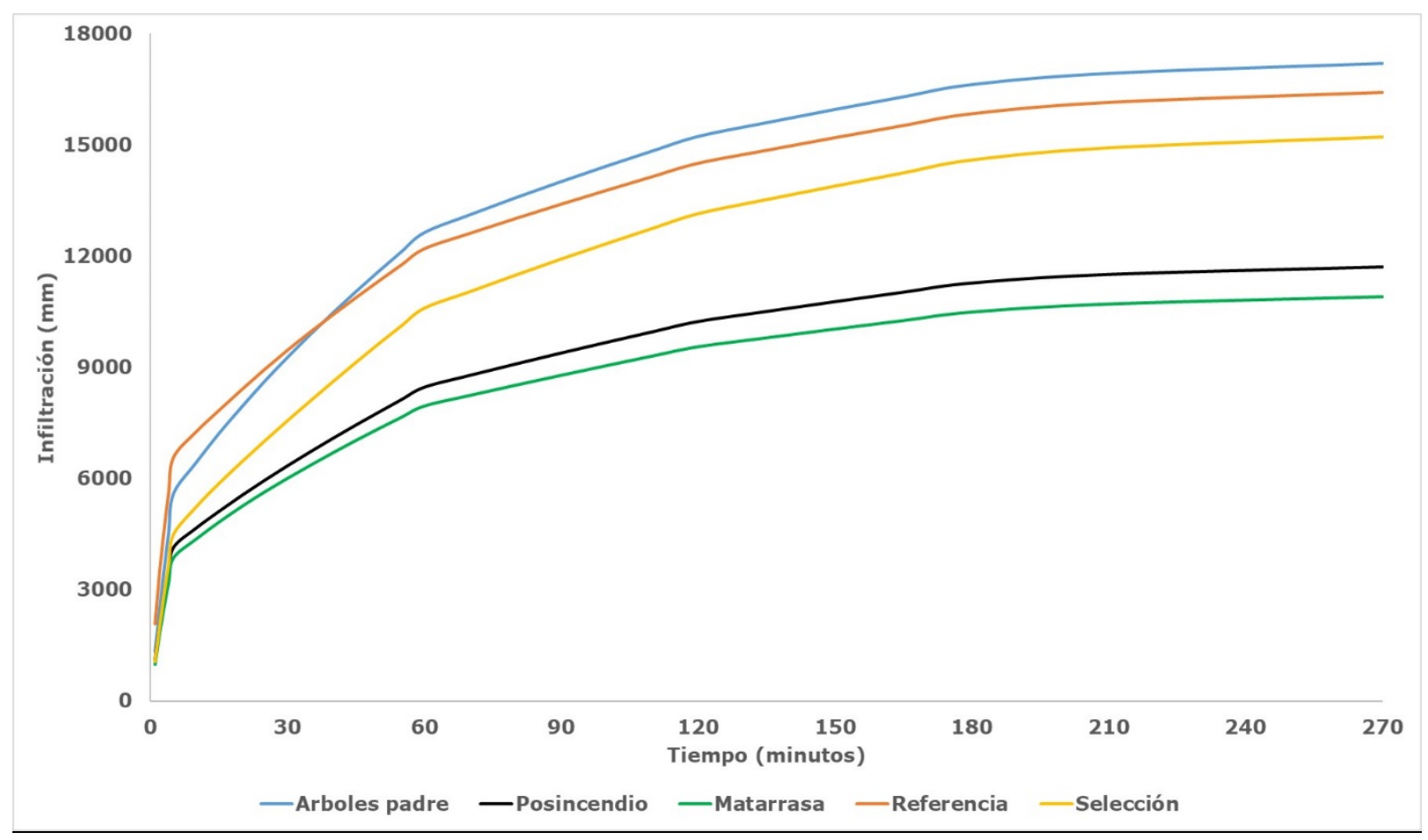

Figura 3. Infiltraciones acumuladas observadas en los rodales silvícolas.

En el Cuadro 3 se resumen los parámetros de los tres modelos ajustados, así como el valor del coeficiente de determinación $\left(R^{2}\right)$, el cual es una medida del grado de bondad de la ecuación empleada. En el mismo Cuadro puede apreciarse que en todos los casos, para los modelos Kostiakov y Lewis-Kostiakov se presentaron mayores valores de $\mathrm{R}^{2}$, además los parámetros asociados a la infiltración inicial ( $F i$ ya) mostraron una tendencia similar a los observados, esto en todos los rodales analizados. 
Cuadro 3. Parámetros de los modelos ajustados y coeficientes de determinación $\left(R^{2}\right)$.

\begin{tabular}{|c|c|c|c|c|c|c|c|c|c|c|c|c|}
\hline \multirow[t]{2}{*}{ Tratamientos } & \multirow[t]{2}{*}{ Medición } & \multicolumn{4}{|c|}{ Kostiakov } & \multicolumn{3}{|l|}{ Horton } & \multicolumn{4}{|c|}{ Lewis-Kostiakov } \\
\hline & & $a$ & $\boldsymbol{b}$ & $R^{2}$ & $\boldsymbol{F b}$ & $\boldsymbol{F i}$ & $\boldsymbol{K}$ & $R^{2}$ & $a$ & $\boldsymbol{b}$ & $\boldsymbol{F b}$ & $\boldsymbol{R}^{2}$ \\
\hline \multirow[t]{3}{*}{ Matarrasa } & $1 \mathrm{er}$ & 1141 & -0.27 & 0.96 & 304 & 993 & -0.05 & 0.92 & 942 & -0.48 & 254 & 0.97 \\
\hline & $2 \mathrm{da}$ & 759 & -0.38 & 0.94 & 172 & 1075 & -0.34 & 0.70 & 719 & -0.74 & 137 & 0.92 \\
\hline & $3 e r$ & 1144 & -0.28 & 0.96 & 300 & 996 & -0.06 & 0.92 & 948 & -0.48 & 251 & 0.97 \\
\hline \multirow[t]{3}{*}{ Árboles Padre } & $1 \mathrm{er}$ & 1191 & -0.34 & 0.95 & 202 & 977 & -0.05 & 0.93 & 1081 & -0.47 & 146 & 0.99 \\
\hline & $2 d a$ & 1671 & -0.21 & 0.98 & 508 & 1392 & -0.02 & 0.95 & 1266 & -0.37 & 453 & 0.98 \\
\hline & $3 e r$ & 248 & -0.23 & 0.97 & 93 & 278 & -0.16 & 0.80 & 189 & -0.61 & 85 & 0.92 \\
\hline \multirow[t]{3}{*}{ Selección } & 1 era & 371 & -0.12 & 0.98 & 111 & 323 & -0.01 & 0.80 & 203 & -0.23 & 168 & 0.99 \\
\hline & $2 d a$ & 1158 & -0.25 & 0.97 & 250 & 926 & -0.02 & 0.95 & 838 & -0.42 & 322 & 0.97 \\
\hline & $3 e r$ & 308 & -0.41 & 0.93 & 133 & 2615 & -422.6 & 0.31 & 184 & -0.48 & 93 & 0.95 \\
\hline \multirow[t]{3}{*}{ Posincendio } & $1 \mathrm{er}$ & 714 & -0.33 & 0.95 & 185 & 908 & -0.27 & 0.74 & 644 & -0.75 & 167 & 0.90 \\
\hline & $2 \mathrm{da}$ & 1510 & -0.32 & 0.95 & 309 & 1263 & -0.05 & 0.76 & 1346 & -0.46 & 215 & 0.89 \\
\hline & $3 e r$ & 1025 & -0.24 & 0.97 & 383 & 1262 & -0.24 & 0.75 & 844 & -0.56 & 299 & 0.94 \\
\hline \multirow[t]{3}{*}{ Rodal de Referencia } & $1 \mathrm{er}$ & 2112 & -0.43 & 0.91 & 413 & 3244 & -0.41 & 0.64 & 2076 & -0.76 & 300 & 0.93 \\
\hline & $2 \mathrm{da}$ & 2214 & -0.43 & 0.92 & 407 & 2973 & -0.32 & 0.71 & 2130 & -0.67 & 263 & 0.96 \\
\hline & 3er & 1379 & -0.27 & 0.96 & 428 & 1387 & -0.13 & 0.82 & 1177 & -0.49 & 300 & 0.97 \\
\hline
\end{tabular}


En la Figura 4, como ejemplo, se exhibe el comportamiento de la tasa de infiltración observada y estimada, a partir de los modelos ajustados de la tercera prueba para las áreas de rodal de Referencia, Selección, Matarrasa y Posincendio; se observa claramente que desde la infiltración inicial, el modelo de Kostiakov tiene un mejor arreglo, a diferencia de Horton el cual no estima de manera adecuada la tasa de infiltración durante los primeros momentos, y después de 30 minutos tienden a sobrestimar la infiltración. En general, ese comportamiento se registró en la mayoría de las pruebas. No obstante, el modelo de Lewis-Kostiakov tuvo un correcto desempeño en el ajuste, cuando se registraron tasas de infiltración inicial bajas $\left(F i<300 \mathrm{~mm} \mathrm{~h}^{-1}\right)$, a diferencia de los modelos de Horton y Kostiakov, los que sobrestimaron y subestimaron la infiltración, respectivamente; lo cual se observa claramente en el área de Selección.
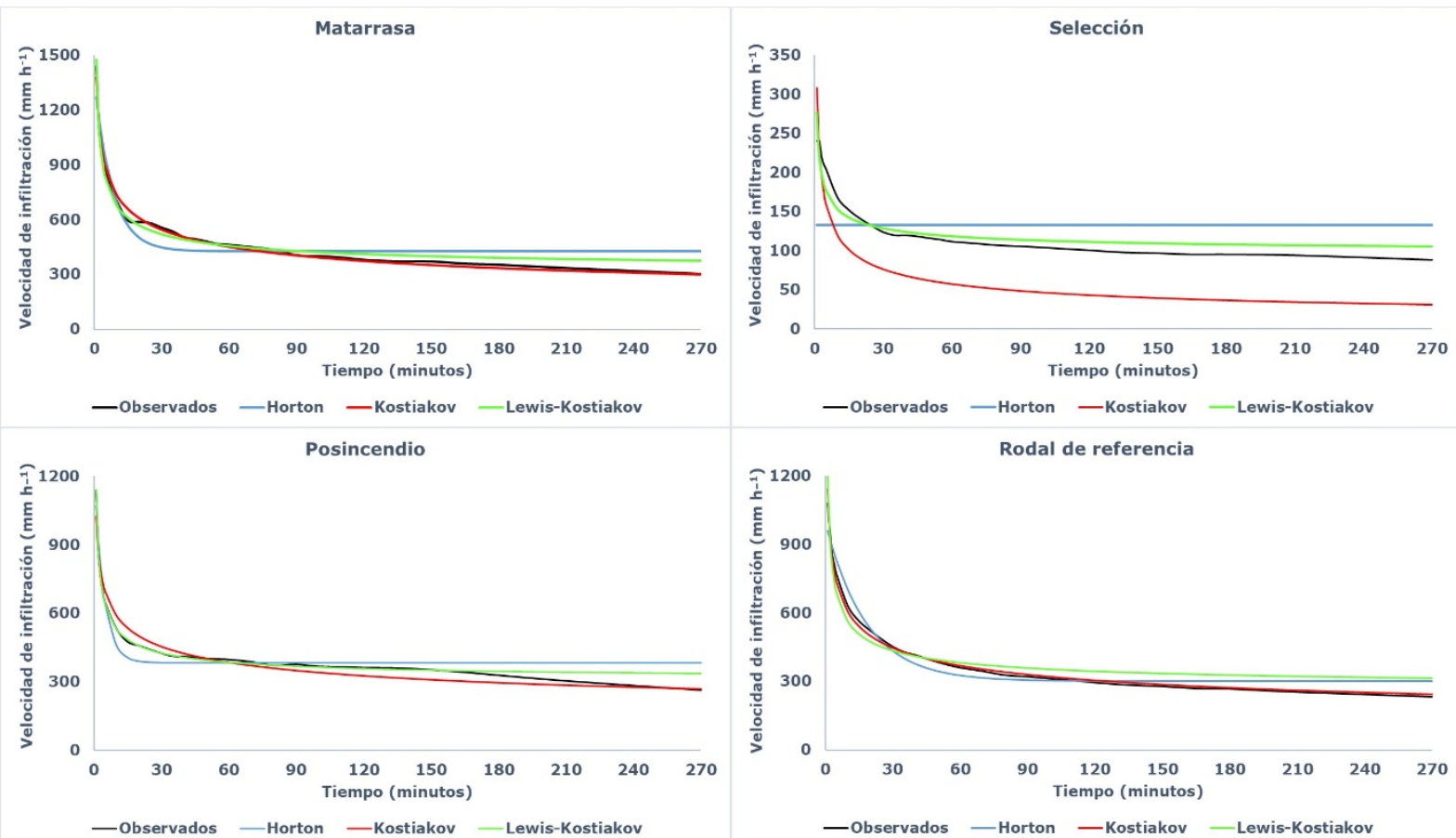

Figura 4. Ajuste de la tasa de infiltración a partir de los modelos de Horton, Kostiakov y Lewis-Kostiakov. 


\section{Discusión}

Di Prima et al. (2017) señalan que la apertura del dosel, debido a la remoción de un cierto número de árboles, es una práctica importante que influye en las relaciones entre el suelo y el agua. Con base en los resultados del presente estudio, las diferencias de la infiltración inicial, básica y acumulada en los rodales están determinadas por el tipo de disturbio o tratamiento silvícola aplicado. Dueñez et al. (2006) y Landini et al. (2007) citan que la intensidad de corta es un factor importante que define los niveles de intercepción de la precipitación, luminosidad, contenidos de humedad y profundidad de materia orgánica en el suelo, etcétera; y por lo tanto, provocan un efecto directo en las propiedades hidráulicas del suelo.

Bens et al. (2007), Wagner et al. (2011) y Archer et al. (2013) indican que la profundidad y la cantidad de materia orgánica contribuyen a mejorar la estructura edáfica e incrementan la capacidad de infiltración, almacenamiento de agua y la conductividad hidráulica; y a partir de los resultados de Fi, Fb y Fa; así como de, las características físicas del suelo y la vegetación que predominan en los rodales estudiados; lo anterior se puede corroborar, principalmente, en el rodal de Matarrasa, cuyas propiedades fueron afectadas de manera importante por la intensidad de corta aplicada, y ello provocó disminuciones en las variables hidrológicas.

Los valores de las variables de infiltración permiten definir lo consignado por Hümann et al. (2011), Marshall et al. (2014) y Archer et al. (2016) quienes sugieren que la etapa de crecimiento del bosque es un factor relevante que determina las características hidrológicas del suelo. Esto concuerda con los resultados del estudio que aquí se documenta, en el que las áreas de Matarrasa y Posincendio son masas forestales que presentan un estado de madurez similar, a diferencia de los rodales de Árboles Padre, Selección y Referencia donde existe arbolado superior.

Respecto a los valores del estadístico $\mathrm{R}^{2,}$ se nota muy poca variación entre el modelo tipo Kostiakov y el de Lewis-Kostiakov, los cuales representan adecuadamente la evolución de la lámina infiltrada en las pruebas de infiltración de los diferentes rodales 
evaluados. Lo anterior se confirma con lo señalado por Návar y Synnott (2000), Weber y Apestegui (2016), Sihag et al. (2017) quienes indican que las mejores predicciones, por lo general, se obtienen con el modelo Lewis-Kostiakov., ya que sus parámetros son más sensibles al tipo de suelos Umbrisol, que se caracteriza por presentar un horizonte superficial grueso y oscuro, insaturado de bases y rico en materia orgánica (Casanova et al., 2007). En relación con lo anterior, disminuciones en la materia orgánica repercuten en la estabilidad de los agregados, al dispersar partículas finas texturales y con ello se favorece la disminución de la porosidad y, en consecuencia, hay decrementos en la infiltración (García-Hernández et al., 2008).

Cabe señalar que la información respecto a los temas hidrológicos y su relación con el manejo forestal es escaza y limitada, con base en ello se resalta la importancia del presente estudio.

\section{Conclusiones}

La tasa de infiltración inicial (2 $080 \mathrm{~mm} \mathrm{~h}^{-1}$ ), básica $\left(297.93 \mathrm{~mm} \mathrm{~h}^{-1}\right.$ ) y acumulada (16 $428.29 \mathrm{~mm}$ ) observadas en el rodal de Referencia evidencian diferencias estadísticas significativas, respecto a los otros rodales analizados; por lo tanto, las modificaciones en la estructura forestal provocan un efecto negativo sobre las variables hidrológicas Fi, Fb y Fa.

Las variables que componen el proceso de infiltración presentan el siguiente orden decreciente:

- Infiltración inicial: Referencia > Árboles Padre> Posincendio > Selección > Matarrasa

- Infiltración básica: Selección > Referencia > Árboles Padre > Posincendio > Matarrasa

- Infiltración acumulada: Árboles Padre $>$ Referencia $>$ Selección $>$ Posincendio $>$ Matarrasa 
Características como el estado de madurez de la vegetación, estructura, composición y las variables edáficas densidad aparente, espesor de capa orgánica y humedad provocan variaciones en las tasas de infiltración en los diferentes rodales analizados. Los resultados del ajuste de modelos, basados en el coeficiente de determinación $\left(R^{2}\right)$, muestran que los de Kostiakov y Lewis-Kostiakov son adecuados para predecir la infiltración en las diferentes condiciones del bosque estudiadas, específicamente, el modelo Lewis-Kostiakov estima mejor la infiltración para las áreas de Selección y Referencia, y en los rodales de Árboles Padre, Matarrasa y Posincendio es mejor descrita por el modelo Kostiakov.

Los parámetros de los modelos de infiltración son de gran importancia para estimar la recarga de los acuíferos, escurrimientos superficiales, erosión del suelo y por ende, facilitan la toma de decisiones con bases de sustentabilidad.

\section{Agradecimientos}

Los autores desean expresar su reconocimiento a la Unidad de Prestación de Servicios Ejidales No. 6 El Salto, A. C., a la Facultad de Ciencias Forestales de la Universidad Autónoma de Nuevo León y al Conacyt por todas las facilidades otorgadas para el desarrollo de la investigación.

\section{Conflicto de interés}

Los autores declaran no tener conflicto de intereses. 


\section{Contribución por autor}

Erik Orlando Luna Robles: desarrollo de la investigación en campo y escritorio, estructura y diseño del manuscrito; Israel Cantú Silva: corrección del manuscrito y análisis estadístico; María Inés Yáñez Díaz: aportación de información analítica al manuscrito; Humberto González Rodríguez: corrección y revisión del documento; José Guadalupe Marmolejo Monsiváis: revisión y correcciones de manuscrito; Silvia Janeth Béjar Pulido: toma y procesamiento de datos del manuscrito.

\section{Referencias}

Archer, N. A. L., M. Bonell, N. Coles, A. M. MacDonald, C. A. Auton and R.

Stevenson. 2013. Características del suelo y relaciones de cobertura del suelo en la conductividad hidráulica del suelo a escala de ladera: una visión hacia el manejo local de inundaciones Journal of Hydrology 497: 208-222. Doi:

10.1016/j.jhydrol.2013.05.043.

Archer, N. A., W. Otten, S. Schmidt, A. G. Bengough, N. Shah and M. Bonell. 2016. Rainfall infiltration and soil hydrological characteristics below ancient forest, planted forest and grassland in a temperate northern climate. Ecohydrology 9(4): 585-600. Doi: $10.1002 /$ eco.1658.

Bens, O., N. A. Wahl, H. Fischer and R. F. Hüttl. 2007. Water infiltration and hydraulic conductivity in sandy cambisols: impacts of forest transformation on soil hydrological properties. European Journal of Forest Research 126(1): 01-109. Doi: $10.1007 /$ s10342-006-0133-7.

Berlanga, V. y H. M. J. Rubio. 2012. Clasificación de pruebas no paramétricas. Cómo aplicarlas en SPSS. REIRE. Revista d'Innovació i Recerca en Educació 5(2):101-113. Doi: $10.1344 /$ reire2012.5.2528. 
Casanova, M., W. Luzio y R. Maldonado. 2007. Correlación entre World Reference Base y Soil Taxonomy para los suelos de la VII Región del Maule de Chile. Revista de la ciencia del suelo y nutrición vegetal 7(2): 14-21. Doi: 10.4067/S0718-27912007000200002.

Collis, G., N. 1977. Infiltration equations for simple soil systems. Water resources research 13(2): 395-403. Doi: 10.1029/WR013i002p00395.

Di Prima, S., V. Bagarello, J. Angulo, R. I. Bautista, A. Cerdà, A. Del Campo and F. Maetzke. 2017. Impacts of thinning of a Mediterranean oak forest on soil properties influencing water infiltration. Journal of Hydrology and Hydromechanics 65(3): 276286. Doi: 10.1515/johh-2017-0016.

Dueñez, A., J. Gutiérrez, J. Pérez y J. Návar. 2006. Manejo silvícola, capacidad de infiltración, escurrimiento superficial y erosión. Terra Latinoamericana 24(2): 233-240.

García-Hernández, M. A., M. A. García-Hernández, I. Castellanos-Vargas, Z. CanoSantana y C. M. Peláez-Rocha. 2008. Variación de la velocidad de infiltración media en seis ecosistemas inalterados. Terra Latinoamericana 26(1): 21-27.

González-Elizondo, M. S., M. González-Elizondo, J. A. Tena-Flores, L. Ruacho-González e I. López-Enríquez. 2012. Vegetación de la sierra madre occidental, México: Una síntesis. Acta Botánica Mexicana (100): 351-403. Doi: 10.21829/abm100.2012.40.

Grego, C. R. y S. R. Vieira. 2005. Variabilidad espacial de propiedades físicas do solo em uma parcela experimental. Revista Brasileira de Ciência do Solo Viçosa 29(2):169-77. Doi: 10.1590/S0100-06832005000200002.

Hillel, D. 1971. Soil and Water. Academic Press. New York, NY, USA. pp. 131-153.

Hümann, M., G. Schüler, C. Müller, R. Schneider, M. Johst and T. Caspari. 2011. Identification of runoff processes-The impact of different forest types and soil properties on runoff formation and floods. Journal of Hydrology 409(3-4): 637-649. Doi: $10.1016 / j . j h y d r o l .2011 .08 .067$. 
Instituto Nacional de Estadística y Geografía (Inegi). 2005. Prontuario de información geográfica municipal de los Estados Unidos Mexicanos. Clave geoestadística. Pueblo Nuevo, Dgo., México. 9 p.

International Business Machines (IBM). 2013. IBM SPSS Statistics for Windows, Version 22.0. IBM Corp. Armonk, New York. E.U.A. n/p.

Landini, A. M., D. Martínez, H. Días, E. Soza, D. Agnes y C. Sainato. 2007. Modelos de infiltración y funciones de pedotransferencia aplicados a suelos de distinta textura. Ciencia del suelo 25(2): 123-131.

Machiwal, D., M. K. Jha and B. C. Mal. 2006. Modelling Infiltration and quantifying spatial soil variability in a Wasteland of Kharagpur, India. Biosystems Engineering 95(4):569-582. Doi: 10.1016/j.biosystemseng.2006.08.007.

Marshall, M. R., C. E. Ballard, Z. L. Frogbrook, I. Solloway, N. McIntyre, B. Reynolds, and H. S. Wheater. 2014. The impact of rural land management changes on soil hydraulic properties and runoff processes: results from experimental plots in upland UK. Hydrological Processes 28(4): 2617-2629. Doi: 10.1002/hyp.9826.

Monárrez, G. J. C., V. G. Pérez, G. C. López, L. M. A. Márquez y M. D. S. G. Elizondo. 2018. Efecto del manejo forestal sobre algunos servicios ecosistémicos en los bosques templados de México. Madera y Bosques 24(2): 1-16. Doi: 10.21829/myb.2018.2421569.

Návar, J. and T. J. Synnott. 2000. Soil infiltration and land use in Linares, NL, Mexico. Terra Latinoamericana 18(3): 255-262.

Pérez, E. G. y A. M. Romance. 2012. Modelación de la infiltración en un campo agrícola de la cuenca del río Chirgua, estado Carabobo, Venezuela. Revista Científica UDO Agrícola 12(2): 365-388.

Pérez O., D. J., J. A. Segovia O., P. C. Cabrera M., I. A. Delgado V. y M. L. Martins P. 2018. Uso del suelo y su influencia en la presión y degradación de los recursos hídricos en cuencas hidrográficas. RIAA 9(1): 1. Doi: 10.22490/21456453.2089. 
Rodríguez-Vásquez, A., A. M. Aristizábal-Castillo y J. H. Camacho-Tamayo. 2008. Variabilidad espacial de los modelos de infiltración de Philip y Kostiakov en un suelo Ándico. Engenharia Agrícola 28(1): 64-75. Doi: 10.1590/S0100-69162008000100007.

Romero S., M. 2016. Pruebas de bondad de ajuste a una distribución normal. Enfermería del Trabajo 6(3): 105-114.

Sahagún-Sánchez, F. J., y H. Reyes-Hernández. 2018. Impactos por cambio de uso de suelo en las áreas naturales protegidas de la región central de la Sierra Madre Oriental, México. Ciencia UAT 12(2): 6-21.

Sihag, P., N. K. Tiwari and S. Ranjan. 2017. Estimation and inter-comparison of infiltration models. Water Science 31(1): 34-43. Doi:10.1016/j.wsj.2017.03.001.

Torres, R., J. M., y S. A. Guevara. 2002. El potencial de México para la producción de servicios ambientales: captura de carbono y desempeño hidráulico. Gaceta Ecológica 63: 40-59.

Turnbull, L., J. Wainwright and R. E. Brazier. 2010. Changes in hydrology and erosion over a transition from grassland to shrubland. Hydrological Processes: An International Journal 24(4): 393-414. Doi: 10.1002/hyp.7491.

Wagner, S., H. Fischer and F. Huth. 2011. Canopy effects on vegetation caused by harvesting and regeneration treatments. European Journal of Forest Research, 130(1): 17-40. Doi: 10.1007/s10342-010-0378-z.

Weber, J. F. y L. Apestegui. 2016. Relaciones entre parámetros de los modelos de infiltración de Kostiakov y Lewis-Kostiakov. Córdoba, Argentina. Tecnología y Ciencias del Agua 7(2): 115-132.

Woerner, M. 1989. Métodos químicos para el análisis de suelos calizos de zonas áridas y semiáridas. Departamento Agroforestal. Facultad de Ciencias Forestales. Universidad Autónoma de Nuevo León. Linares, N.L., México. 105 p. 
Yáñez-Díaz, M. I., I. Cantú-Silva, H. González-Rodríguez and L. Sánchez-Castillo. 2019. Effects of land use change and seasonal variation in the hydrophysical properties in Vertisols in northeastern Mexico. Soil Use and Management 35(3):10. Doi: $10.1111 /$ sum. 12500.

Zhang, J., T. Lei, L. Qu, P. Chen, X. Gao, C. Chen and G. Su. 2017. Method to measure soil matrix infiltration in forest soil. Journal of Hydrology 552: 241-248. Doi: $10.1016 /$ j.jhydrol.2017.06.032.

Zúñiga, J., E. Martínez, C. Navarrete, J. D. J. S. Luna, D. M. Ayala, y B. Mejía. 2018. Análisis ecológico de un área de pago por servicios ambientales hidrológicos en el ejido La Ciudad, Pueblo Nuevo, Durango, México. Investigación y Ciencia73: 27-36.

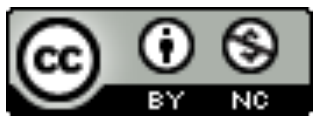

Todos los textos publicados por la Revista Mexicana de Ciencias Forestales -sin excepción- se distribuyen amparados bajo la licencia Creative Commons 4.0 Atribución-No Comercial (CC BY-NC 4.0 Internacional), que permite a terceros utilizar lo publicado siempre que mencionen la autoría del trabajo y a la primera publicación en esta revista. 